\title{
Cashless, Tech-Savvy Future Surgeon
}

\author{
Nayan Agarwal ${ }^{1} \cdot$ Chintamani $^{2}$
}

Published online: 4 June 2016

(C) Association of Surgeons of India 2016

Surgeons have always been depicted as heroes in various novels, movies and stories [1]. We cannot forget the quintessential hero walking out of an operation theatre with his blood-soaked gloves being looked at by all as an avatar of the divine. They have excelled not only in surgery but have left bold strokes on the canvas of domains as diverse as writing detective stories (Sherlock Holmes) to childhood fantasies (Jurassic Park). Apart from all this, surgeons have left their footprints on the political landscape too, building cities and nations. Justus Ohage, the first surgeon to do a cholecystectomy in the USA was ostracised by the surgical community for doing cholecystectomy when cholecystostomy was still the gold standard of gallstone disease management. Outside surgery, he became the most famous public health officer of Minnesota, still remembered for his contribution in building the modern city.

Today, India is undergoing a rapid transition with a fastgrowing economy driven by consumerism. Payment of money, through various modalities, is also increasing accordingly, with more and more consumers adopting newer modes apart from cash like plastic money and electronic bank transfers. The government is evolving strategies to encourage cashless transactions for various benefits like more accountability, less economic burden of physical currency and curbing generation of black money. In this context, surgeons too can contribute to this ideal. Doctors are inherently philanthropic and, as such, never focus on earning money as a primary goal. But still, exchange of money in the form of private consultations and

Chintamani

drchintamani7@gmail.com

UCMS and GTB Hospital, Delhi, India

2 VMMC and Safdarjung Hospital, New Delhi, India hospital bills is both a necessity and the norm nowadays. Consultations, which are an important preoperative resource for the surgeon, are usually paid for in cash by the patient. According to available evidence, new payment methodologies are likely to facilitate the appropriate use of this important resource [2]. With the growth of technology, 'e-consultation' is quickly becoming a reality with its own benefits for the patients, primary healthcare provider, specialist and even the healthcare system [3]. Although the use of credit cards is now ubiquitous among all generations, its use, possible with a mandated accompanying machine, is still not as easy, feasible or viable for the individual practitioner. This is where the use of a truly digital means of payment, i.e. Mobile wallets (mWallets) like paytm (www.paytm.com) and FreeCharge (www. freecharge.com), can be of great application to both the surgeon and the patient. The younger generation today is frequently using such applications to pay for services ranging from shopping to food to even taxis on the road. For a surgeon, the use of such mWallets provides for instantaneous and accountable receipt of the consultation fee without the need for any physical exchange of money, especially in those situations where the availability of cash may be compromised for any reason. No one checks on their wallets in a rush, but a smartphone today has become a tool for survival and, as such, is available on the person at all times.

With over a billion mobile subscribers in the country, the appeal of such smartphone-based banking applications is a 'no-brainer'. For example, if one was to use paytm regularly, the doctor and the patient need only to sign up with their mobile number and optionally complete Know Your Customer (KYC) formalities to enable transactions of up to Rs. 1 Lakh ( US\$1500) per month. Even without KYC, transactions of up to Rs. 10,000 per month (Rs. 1.2 Lakh per annum) can be carried out without any sort of paperwork involved. Moreover, the money accrued in the mWallet can 
be transferred to a conventional bank account from the mobile app itself, apart from being usable for various online transactions like a virtual debit card, without any sort of transaction charges at any point. e-Consultation is fast becoming a reality and the use of SMS and WhatsApp among other communication methods to seek medical consultation warrants the utilisation of these mWallets by the surgeon to enable traceable and accountable receipt of payments. Cashless medical insurance has already become the norm in today's world, and a similar, effective solution for medical consultation has come up in the form of mWallets.

In this technology-driven world, the surgeon has been a leader in the adoption of technology, albeit with scepticism. Advanced laparoscopic surgery has become the flag bearer of the fusion of medical sciences with technology. The surgeon as a tech leader has fused his knowledge of innovation, molecular sciences, anatomy, genetics, information technology and nanotechnology into 'imagineering'-driven improvements in outcomes for the community as a whole $[4,5]$. Even with the advancements in minimally invasive surgical technologies, faster and faster convalescence for the patient is being pursued. A modern surgeon is tirelessly working on evolving fast-track protocols to minimise the side effects of surgery [6]. Technology is not a strange partner to the surgeon; it is a double-edged sword [7]. While it has enabled surgeon to master minimally invasive therapies, at the same time, it has put additional demands on the surgeon by tech-enabled patient seeking a personalised, 'all-in' approach. So, it is better to adopt any technology which is being used by the society at large including those which make the patient feel more involved with the surgeon, directly or indirectly. The use of direct, mobile-based payment solutions contributes to this feeling.

Thus, the benefits of using such mWallets not only simplify the hassles associated with cash-based payment for the surgeon and the patient but would also contribute to promoting a cashless future. This will help reduce not only the costs to the government and eventually tax-paying citizens of India but also the inherent 'carbon footprint' of paper currency, hence conserving the environment [4]. Being completely traceable and accountable, the potential of mWallet payments to reduce the misuse of money is a great advantage with far-reaching consequences in the control of black money, illegal trade and tax evasion. Cashless financial transactions have a strong potential to contribute in eradication of mis-endeavours like fake currency and drug and terror financing which proliferate in the presence of paper currency. With smartphones becoming a basic necessity and a tool of change, it is fair to say that ubiquitous, smartphone-based payments will be the next revolution in the rapidly changing economic scenario. Surgeons are natural leaders, and taking the lead in creating the cashless future of our country should come to us naturally. Hence, a tech-savvy surgeon of the future can contribute to personal and professional growth, social advancement, environmental conservation and geopolitical stability.

\section{References}

1. Agarwal BB, Chintamani, Ali K, Goyal K, Mahajan KC (2012) Innovations in endosurgery-journey into the past of the future: to ride the SILS bandwagon or not? Indian J Surg 74:234-241

2. Thilen SR, Wijeysundera DN, Treggiari MM (2016) Preoperative consultations. Anesthesiol Clin 34:17-33

3. Liddy C, Rowan MS, Afkham A, Maranger J, Keely E (2013) Building access to specialist care through e-consultation. Open Med 7:e1-e8

4. Agarwal BB (2008) Journey of the carbon-literate and climateconscious endosurgeon having a head, heart, hands, and holistic sense of responsibility. Surg Endosc 22:2539-2540

5. Agarwal BB, Nanavati JD, Agarwal N, Sharma N, Agarwal KA, Manish K, Saluja S, Agarwal S (2016) Biomolecular inflammatory response to surgical energy usage in laparoscopic surgery: results of a randomized study. Surg Endosc 30:1733-1741

6. Agarwal BB, Chintamani, Agarwal S (2015) Fast track surgeryminimizing side effects of surgery. Indian J Surg 77:753-758

7. Agarwal BB (2010) Umbilicus, navel, belly button - Vitruvian guide for esthetic cosmetics: a Da Vinci code for beautiful informed consent. Surg Endosc 24:236-238 